\title{
Electrical stimulation-a mapping system for hand dysfunction in tetraplegia
}

\author{
Ines Bersch ${ }^{1,2} \cdot$ Sabrina Koch-Borner ${ }^{1} \cdot$ Jan Fridén ${ }^{1,3}$
}

Received: 19 June 2017 / Revised: 1 November 2017 / Accepted: 2 November 2017 / Published online: 22 January 2018

(c) International Spinal Cord Society 2018

\begin{abstract}
Study design Retrospective data analysis

Objectives To define the distribution of the motor points and excitability of the key wrist and finger actuators in order to detect upper (UMN) and lower motor neuron (LMN) lesions potentially influencing the development of a tenodesis grasp. Setting A rehabilitation centre for spinal cord injuries, Nottwil, Switzerland.

Methods Forearm muscles of 32 patients with tetraplegia (AIS A-D) were tested bilaterally with electrical stimulation (ES) to differentiate whether UMN or LMN was present. For testing, a standardised mapping was developed. All patients underwent the same positioning schedule.

Results Sixteen hands developed a tenodesis grasps, 24 hands showed neither shortening nor tightening of the finger flexors. Two patients developed unilateral tenodesis grasp and showed no tightening of the finger flexors on the contralateral hand. Seven patients developed tenodesis grasps symmetrically and bilaterally, whereas one maintained an essentially open hand without tightening of the finger flexors. All hands that developed a tenodesis grasp showed a LMN lesion of the M. extensor digitorum communis (EDC). The frequency of the tenodesis grasp differed significantly between the groups with and without intact reflex arc $(p<0.0001)$.

Conclusion Surface ES may serve as a diagnostic tool to detect an UMN or LMN lesion of the key actuator muscles affecting the tenodesis grasp. These findings provide information that is essential for the choice of treatment to optimise function of the tetraplegic hand.
\end{abstract}

\section{Introduction}

For many patients with tetraplegia after a spinal cord injury at $\mathrm{C} 5-\mathrm{C} 7$ levels, regaining hand function is the most important goal during rehabilitation. A common and widely used treatment aim is to establish a functional tenodesis grasp. In order to reach this goal, the hand is positioned so that the finger flexors will tighten over time and the wrist extension will enhance the so called "functional hand." The tenodesis grasp manifests as active or passive. The major

$\triangle$ Ines Bersch

ines.bersch@paraplegie.ch

1 Swiss Paraplegic Centre, Nottwil, Switzerland

2 Institute of Clinical Sciences, Department of Orthopaedics at the University of Gothenburg, Gothenburg, Sweden

3 Centre for Advanced Reconstruction of Extremities (CARE), Tetraplegia, Hand Surgery, Sahlgrenska University Hospital and the Sahlgrenska, Academy at the University of Gothenburg, Göteborg, Sweden difference lies in the presence of a voluntary wrist extension. An active functional tenodesis grasp entails a sufficient closure of all fingers. Typically, the finger joints should be flexed $90^{\circ}$ in the metacarpophalangeal (MCP) and proximal interphalangeal (PIP) and $0^{\circ}$ in the distal interphalangeal (DIP) joints during an active wrist extension. In case of a passive tenodesis grasp the wrist extension is performed passively by a supination of the forearm. Thereby, the wrist moves by gravity into a wrist extension and the fingers will close. Responsible for this mechanism of hand closure is the volitional shortening and tightening of the flexor digitorum superficialis muscle (FDS) caused by a hand positioning and splinting schedule as well as the prevention of MCP and PIP contractures [1,2]. The latter is performed by passive finger flexion in response to wrist extension [3]. For opening of the hand, the wrist needs to be actively or passively flexed so that the passive closure of the fingers will be released (Fig. 1a, b).

Clinical observations have indicated that the outcome of positioning, splinting and passive mobilisation of the finger joints is frequently unpredictable and unsuccessful. Doll 
Fig. 1 a Hand opening performed by release of tenodesis grasp, b Hand closure performed by tenodesis grasp (Colour figure online)

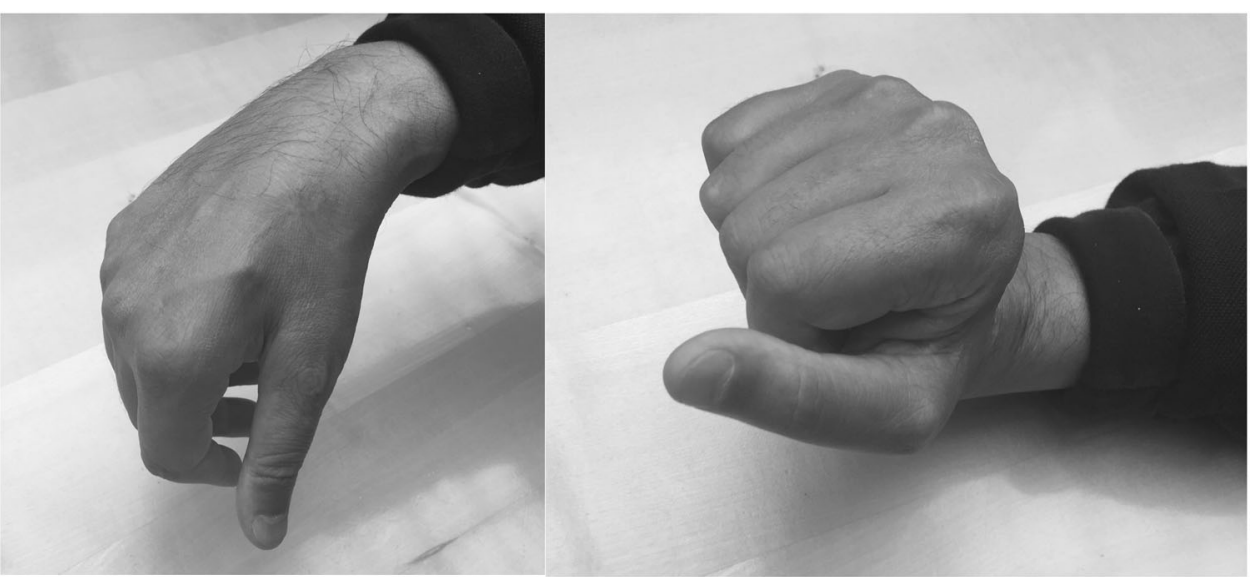

and co-workers reported a $50 \%$ rate of success in establishing the functional tenodesis grasp [1]. Harvey et al. [4] found similar results by splinting the thumb in a shortened position to minimise extensibility of the flexor pollicis longus (FPL) muscle. A functional tenodesis grasp is described as the closure of the hand by active or passive wrist extension including the closure of the PIP of the thumb on the level of the DIP of the index finger $[1,3,5]$.

It is well-known that the degree of neurological recovery as well as spasticity interfere with the attempts to develop a functional hand based on tenodesis effect [6]. Furthermore, presence of an intact upper (UMN) or lower (LMN) motor neuron in the forearm muscles may influence the development of the hand posture [4]. The UMN and LMN are usually intact above the lesion. Below the lesion, there is an UMN lesion with intact reflex arcs and an intact LMN, whereas at the level of lesion the LMN may be partially or completely damaged [7]. Mulcahey et al. [8] evaluated the LMN integrity for the muscles for grasp and release as well as for the wrist extensor. They showed in 23 tested tetraplegic arms an intact LMN in $83 \%$ for the muscles of grasp and release and in $74 \%$ of the wrist extensors. The consequence of a damaged LMN is a structural and functional reorganisation of muscle properties in comparison to an UMN lesion. Denervation atrophy implies a decrease in muscle fibre diameters and a partial transformation into connective and adipose tissue [9, 10]. In tetraplegia, the forearm muscles present different forms of damage and adaptation. The differentiation between an UMN and LMN can be readily performed through electrical stimulation (ES) via surface electrodes and has been advocated as a standard assessment after cervical SCI [11, 12].

The first aim of the retrospective data analysis was to demonstrate that an ES mapping system of the forearm can be used to detect an UMN and LMN lesion of the key actuator muscles affecting the tenodesis grasp. The second aim was to analyse if a relation between the motor neuron lesion in the tested muscles and the final hand posture could be detected.

\section{Methods}

Data from patients who consented for retrospective data analysis were included in the study. Thirty-two inpatients during rehabilitation with cervical SCI (ASIA impairment scale (AIS) A-D, age 44.9 \pm 19.2 (mean \pm SD) years, time since lesion $1.4 \pm 3($ mean $\pm \mathrm{SD})$ years were included in the study (Table 1). The muscles of the patients' forearms were tested by ES to differentiate whether an UMN or LMN was present. In the rehabilitation centre, where all data were collected, ES serves as a standard assessment for all tetraplegic patients during rehabilitation. All patients underwent the identical hand positioning protocol. The AIS was performed as a standard examination by a neurologist. All patients underwent neurological examination within the first 4 weeks, after 3 and 6 months and before discharge after 8 to 9 months.

\section{Hand positioning}

The hand positioning schedule was initiated within $48 \mathrm{~h}$ after injury. The duration of the schedule ( 6 or 8 weeks) was depending on whether the desired position had been achieved or not. Shortening of the finger flexors yielded as sufficient when the finger pads of finger II-IV touched the palmar side of the hand during active or passive wrist extension.

During daytime, the positioning for the hand was performed in $30^{\circ}$ wrist extension, $90^{\circ}$ flexion in the MCP and PIP joints and $0^{\circ}$ in the DIP joints. This position was secured with a splint in combination with taping of the fingers in case of an inactive $M$. extensor carpi radialis (ECR) (radial wrist extensors M. extensor carpi radialis brevis and longus- ECRB and ECRL). In case of an active ECR exceeding British Medical Research Council Scale (MRC) [13] strength grade 3 the fingers were kept in the above described fist position with a glove. Splints and gloves were only removed during active or passive training 
Table 1 Patient characteristics

\begin{tabular}{|c|c|c|c|c|c|c|}
\hline ID & Gender & Age & $\begin{array}{l}\text { Level of } \\
\text { lesion }\end{array}$ & AIS & $\begin{array}{l}\text { ICSHT } \\
\text { motor score }\end{array}$ & $\begin{array}{l}\text { Time since } \\
\text { injury (years) }\end{array}$ \\
\hline 1 & $\mathrm{~m}$ & 22.6 & C5 & A & 1 & 2.5 \\
\hline 2 & $f$ & 28.8 & C6 & B & 3 & 2 \\
\hline 3 & $\mathrm{~m}$ & 30.5 & C5 & A & 1 & 1.8 \\
\hline 4 & $\mathrm{~m}$ & 46.1 & C6 & B & 3 & 17 \\
\hline 5 & $\mathrm{~m}$ & 49.4 & $\mathrm{C} 2$ & $\mathrm{C}$ & 0 & 1.6 \\
\hline 6 & $\mathrm{~m}$ & 17.8 & C5 & A & 1 & 1.8 \\
\hline 7 & $\mathrm{~m}$ & 19.2 & C5 & A & 1 & 2 \\
\hline 8 & $\mathrm{~m}$ & 18.8 & $\mathrm{C} 4$ & A & 0 & 1.4 \\
\hline 9 & $\mathrm{~m}$ & 65.2 & C5 & A & 1 & 1 \\
\hline 10 & $f$ & 33.0 & $\mathrm{C} 4$ & B & 0 & 4.9 \\
\hline 11 & $\mathrm{~m}$ & 18.9 & C6 & A & 3 & 1.8 \\
\hline 12 & $\mathrm{~m}$ & 48.2 & C5 & $\mathrm{C}$ & 1 & 0.6 \\
\hline 13 & $\mathrm{f}$ & 73.6 & $\mathrm{C} 4$ & $\mathrm{C}$ & 0 & 0.6 \\
\hline 14 & $\mathrm{f}$ & 45.8 & $\mathrm{C} 3$ & $\mathrm{C}$ & 0 & 0.6 \\
\hline 15 & $\mathrm{~m}$ & 19.3 & $\mathrm{C} 4$ & $\mathrm{C}$ & 1 & 0.6 \\
\hline 16 & $\mathrm{~m}$ & 51.5 & $\mathrm{C} 3$ & $\mathrm{C}$ & 0 & 0.5 \\
\hline 17 & $\mathrm{~m}$ & 55.1 & C5 & $\mathrm{C}$ & 2 & 0.5 \\
\hline 18 & $\mathrm{~m}$ & 76.1 & C5 & B & 1 & 0.4 \\
\hline 19 & $\mathrm{~m}$ & 41.8 & C5 & $\mathrm{D}$ & 5 & 0.4 \\
\hline 20 & $\mathrm{~m}$ & 59.5 & C6 & B & 3 & 0.3 \\
\hline 21 & $\mathrm{~m}$ & 37.1 & $\mathrm{C} 7$ & A & 2 & 0.3 \\
\hline 22 & $\mathrm{~m}$ & 62.0 & $\mathrm{C} 3$ & $\mathrm{C}$ & 1 & 0.2 \\
\hline 23 & $\mathrm{~m}$ & 64.7 & C6 & $\mathrm{D}$ & 0 & 0.3 \\
\hline 24 & $\mathrm{~m}$ & 57.1 & $\mathrm{C} 4$ & $\mathrm{D}$ & 4 & 0.2 \\
\hline 25 & $\mathrm{~m}$ & 60.0 & $\mathrm{C} 2$ & $\mathrm{D}$ & 5 & 0.2 \\
\hline 26 & $\mathrm{~m}$ & 29.8 & C5 & B & 1 & 0.2 \\
\hline 27 & $\mathrm{f}$ & 76.4 & $\mathrm{C} 4$ & A & 1 & 0.3 \\
\hline 28 & $\mathrm{~m}$ & 27.9 & C5 & B & 5 & 0.1 \\
\hline 29 & $\mathrm{~m}$ & 74.4 & $\mathrm{C} 4$ & $\mathrm{C}$ & 1 & 0.1 \\
\hline 30 & $\mathrm{~m}$ & 60.0 & $\mathrm{C} 2$ & $\mathrm{D}$ & 5 & 0.2 \\
\hline 31 & $\mathrm{~m}$ & 21.2 & C6 & $\mathrm{C}$ & 3 & 0.1 \\
\hline 32 & $\mathrm{~m}$ & 44.7 & C5 & A & 1 & 0.1 \\
\hline
\end{tabular}

$m$ male, $f$ female, AIS ASIA Impairment Scale, ICSHT motor score International Classification for Surgery of the Hand in Tetraplegia

in physio- or occupational therapy. The therapy, 5 times a week for $25 \mathrm{~min}$ each included passive mobilisation and task-specific training depending on the patients' skills. Task-specific training included grooming, eating and grasping. The sequence of the training, regarding dose and intensity, was based individually on each patient's strength and endurance referring to the principles of training physiology [14]. Three sets were performed, with a rest of 1 min in between the sets. The number of repetition in each set varied from 10 to 40 for each movement in case of endurance training and 10 to 15 in case of muscle strength [15].
During night, a tenodesis splint secured $0^{\circ}$ wrist extension, $90^{\circ}$ flexion in the MCP and $0^{\circ}$ in the PIP and DIP joints. This protocol was applied for 6 to 8 weeks.

\section{Topographical definition of motor point position}

In order to enable reproducible and simple testing, the stimulation points were mapped for extensor carpi ulnaris (ECU), extensor carpi radialis longus (ECRL), extensor digitorum communis (EDC), extensor pollicis longus (EPL) and abductor pollicis longus (APL) muscles. A diagonal line was drawn on the participants' forearm. The line stretches from the most prominent part of the lateral epicondyle to the most prominent part of radial styloid (A-B). This line served as a landmark to detect the stimulation points of the muscles. The location of the stimulation points of each muscle in relation to line A-B was based on and defined after repetitive measurements in clinical practice. Therefore, the mapping was first tested in able body people for its reproducibility before applying on patients with tetraplegia. The baseline was the calculation of the length of the distance A-B of 20 ( 8 male, 12 female) able body adults. The mean distance A-B yielded $27.1 \pm 1.7$ (mean \pm $\mathrm{SD}) \mathrm{cm}$. This mean length served as the reference length. The distances on line $\mathrm{A}-\mathrm{B}$ and $1 \mathrm{a}-1$ and $2 \mathrm{a}-2$ of the stimulation points 1 to 4 were generated in relation to the length of A-B. Accordingly, the relations of the stimulation points were determined and as described calculated. This map was applicable to all tested forearms.

The stimulation points were calculated and defined as follows (Fig. 2):

ECU: distance $\mathrm{A}-1 \mathrm{a}=$ distance $\mathrm{A}-\mathrm{B} \times 0.18$ and distance $1 \mathrm{a}-1=$ distance $\mathrm{A}-\mathrm{B} \times 0.15$, $\mathrm{ECRL}:$ distance $\mathrm{A}-2 \mathrm{a}=$ distance $\mathrm{A}-\mathrm{B} \times 0.3$ and distance $2 \mathrm{a}-2=$ distance $\mathrm{A}-\mathrm{B} \times$ 0.07, EDC: distance A-3 $=$ distance $\mathrm{A}-\mathrm{B} \times 0.44$, EPL: distance $\mathrm{A}-4=\mathrm{A}-\mathrm{B} \times 0.6$, APL: distance A-5 = distance A$\mathrm{B} \times 0.62$.

The distance 1a-1 directs perpendicular from line A-B in ulnar direction, whereas distance $2 \mathrm{a}-2$ directs perpendicular from line $\mathrm{A}-\mathrm{B}$ in radial direction.

\section{Electrical stimulation protocol}

For testing, the EMPI 300 PV portable neuromuscular stimulator was used. The stimulation was performed with a duration of $300 \mu \mathrm{sec}$ at a frequency of $35 \mathrm{~Hz}$. The amplitude ranged from 20 to $40 \mathrm{~mA}$. One self-adhesive electrode $32 \mathrm{~mm}$ (Axelgaard Pals, Lystrup, Denmark) was placed over the lateral epicondyle to excite all extensor muscles as well as all muscles innervated by the radial nerve. The corresponding electrode was a pen electrode with a diameter of $0.5 \mathrm{~cm}$ that was placed over the motor point of each selected muscle (Fig. 3). 
Fig. 2 Landmark line A-B lateral condyle to radial styloid including distances $\mathrm{A}-1 \mathrm{a}, 1 \mathrm{a}-1$, A-2a, 2a-2, A-3, A4 and A5 with stimulation points of 1 ECU, 2 ECR, 3 EDC, 4 FPL, 5 APL (Colour figure online)

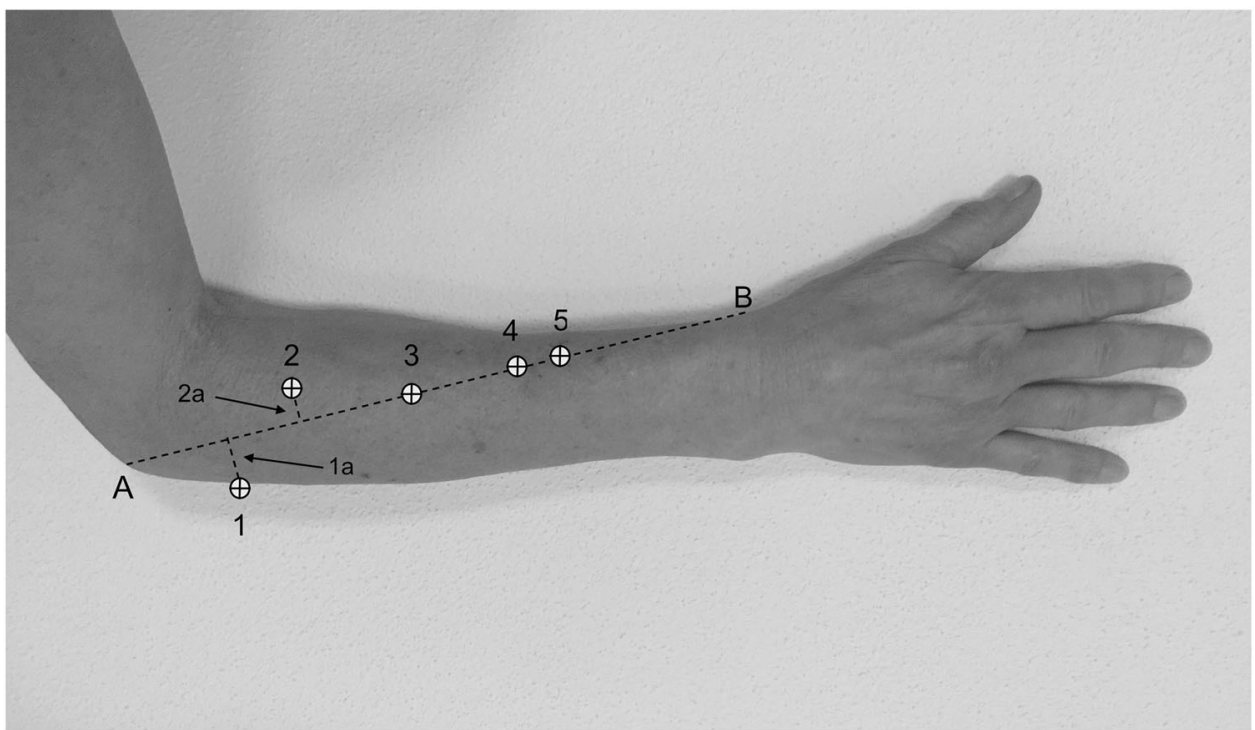

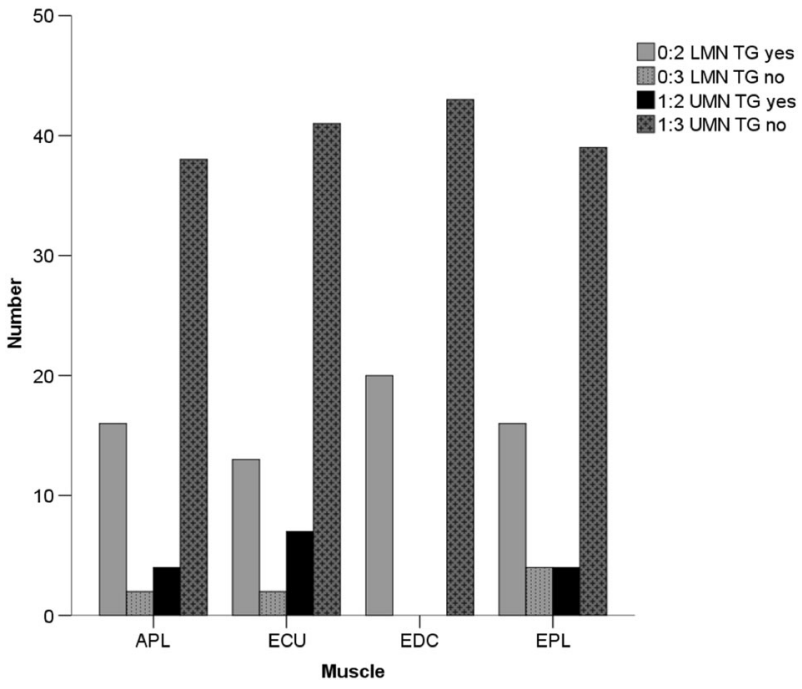

Fig. 3 The distribution of LMN lesion (0) and an UMN lesion [1] in combination with the presence of a tenodesis grasp $(2=$ yes, $3=$ no) of the tested muscles is illustrated. To illustrate the combination of the presence or absence of a tenodesis grasp, respectively, a LMN or UMN lesion a numerical code was chosen. The characters 0 and 1 refer to the type of lesion, whereas 2 and 3 relate to the tenodesis grasp. For example, 0:2 means a LMN lesion with the presence of a tenodesis grasp. Abbreviations: APL M. abductor pollicis longus, ECU M. extensor carpi ulnaris, EDC M. extensor digitorum communis, EPL M. extensor pollicis longus, 0:2 LMN TG yes lower motor neuron lesion and tenodesis grasp, 0:3 LMN TG no lower motor neuron lesion no tenodesis grasp, 1:2 UMN TG yes upper motor neuron lesion tenodesis grasp, 1:3 UMN TG no upper motor neuron lesion no tenodesis grasp

To guarantee the accuracy reproducibility of ES testing three experienced therapists (two physiotherapists and one occupational therapist) performed the stimulation. They were trained and supervised by the person who developed the above described mapping. A muscle yielded as innervated in case of $\geq 3$ MRC during ES, partially innervated in case of $<3 \mathrm{MRC}$ and denervated if no muscle contraction could be provoked.

\section{Statement of ethics}

We certify that all applicable institutional and governmental regulations concerning the ethical use of human volunteers were followed during the course of this research. Patients who did not consent to retrospective data analyses were excluded.

\section{Statistical methods}

Data are presented as mean and range or SD. The location of motor points was determined by topographical description and expressed as an equation where the stimulation points were calculated in relation to the mean length

A-B of the reference group (able body subjects) and confidence interval (CI) that was determined at $95 \%$ level. The Chi-Square test was used to verify the observed frequency distribution from the theoretical distribution.

\section{Results}

The data of 32 patients (63 hands) were analysed. Five patients $(15.6 \%)$ showed a LMN lesion on both sides on the ECU, three patients $(9.4 \%)$ on one side. Eight patients (25\%) had a LMN lesion on both sides and four patients $(12.5 \%)$ on one side on the EDC. Regarding the two ES tested thumb muscles, seven $(21.9 \%)$ patients showed a LMN bilaterally on the EPL and six (18.7\%) on one side. Seven patients $(21.9 \%)$ had a LMN on the APL on both sides, whereas in four patients $(12.5 \%)$ a LMN was present on one side. In five patients partially innervated muscles were found (Fig. 4). 


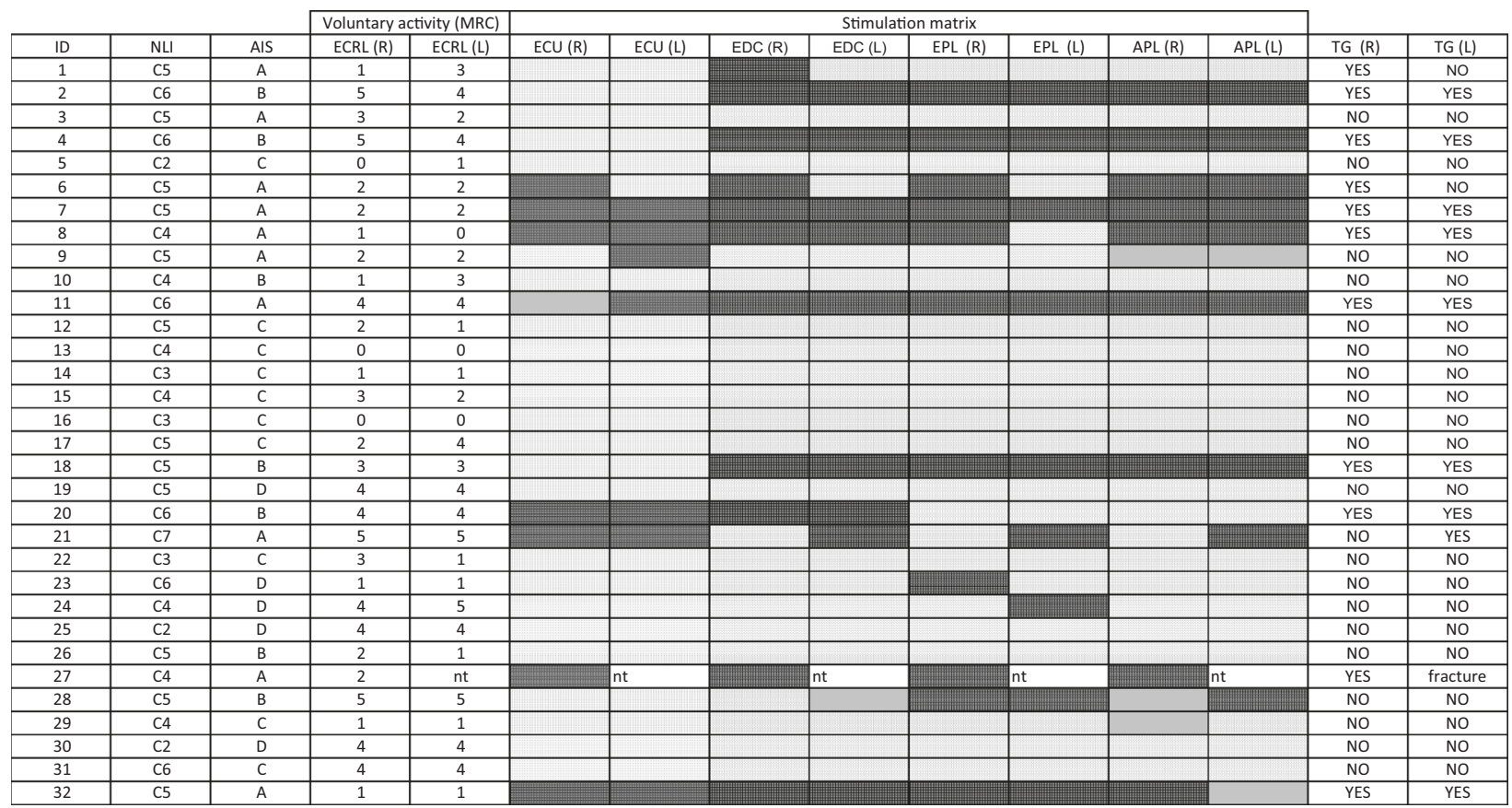

Fig. 4 Stimulation matrix. $N L I$ neurological level of injury, AIS ASIA impairment scale, $M R C$ British medical research council scale, $E C R L>M$. extensor carpi radialis longus, $E C U$ M.extensor carpi ulnaris, $E D C$ M.extensor digitotrum communis, $E P L$ M.extensor pollicis longus, $A P L$ M. abductor pollicis longus, $T G$ tenodesis grasp, $Y E S$ tenodesis grasp, $N O$ no tenodesis grasp, light spotted innervated (upper motor neuron lesion), grey partially denervated, dark checkered denervated (lower motor neuron lesion)

Twenty hands (31.7\%) of 63 developed a tenodesis grasp. Four patients $(12.5 \%)$ developed an unilateral tenodesis grasp and showed no shortening of the finger flexors on the contralateral hand. Of the remaining 28 patients, 8 (28.6\%) developed tenodesis grasps symmetrically and bilaterally (Fig. 4). The hand positioning schedule lasted in 9 patients (28.1\%) 6 weeks and in 21 patients (65.6\%) 8 weeks.

The stimulation points were identified and defined (Table 2). All hands that developed a tenodesis grasp were not excitable by electrical nerve stimulation on the EDC, including one patient, who had a partially innervated EDC on one side. The frequency of the tenodesis grasp differed significantly between the groups with an UMN lesion and LMN lesion $(p<0.0001)$ (Fig. 3).

\section{Discussion}

The present study showed that the type of lesion-UMN or LMN-in the forearm muscles seems to influence the position of the hand and its balance in patients with tetraplegia. Furthermore, the described mapping of functionality with ES of defined muscles in the forearm (ECU, ECRL, EDC, EPL and APL) can be applied easily in clinical routine by clinicians and therapists. The knowledge about the presence of a LMN or UMN lesion of the key actuators affecting the tenodesis grasp might help in the understanding of the expected outcome of the hand positioning protocol.

All patients who had a LMN lesion of the EDC developed a functional tenodesis grasp. In contrast, the patients who had an UMN lesion of the EDC maintained hands lacking shortening of the finger flexors. Both patients' groups, with an UMN and LMN lesion, underwent the same hand positioning schedule as described before. Interestingly the hands of LMN lesion group of the EDC showed a relaxed position of the hand from the beginning of the positioning of the tenodesis grasp, whereas the UNM lesion group showed more extended fingers mainly in the MCP and PIP joints. Our observations coincide with data reported by Harvey et al. $[4,16]$. They observed that the extensibility of the FPL cannot be decisively reduced by night time splinting in a shortened positon of the thumb muscle. Furthermore, they proposed that muscles with an UMN lesion are less responsive to immobilisation in a shortened position [4]. The latter fact is consistent with our detection that the type of lesion in the EDC might be responsible for the development of the hand position. In a LMN lesion of the EDC, a functioning reflex arc is absent and consequently the structure of the muscle will transform into noncontractile tissue as collagen and fat [17]. Hence, the finger flexors have no active antagonists and and this may lead to tightness of the flexor muscles. 
Table 2 Localisation of stimulation points

\begin{tabular}{lccc}
\hline Muscle & $\begin{array}{c}\text { Muscle I } \\
\text { coordinates on } \\
\text { line A-B }\end{array}$ & $\begin{array}{c}\text { Muscle } \\
\text { coordinates } \\
\text { ulnar-radial to } \\
\text { line A-B }\end{array}$ & $\begin{array}{c}\text { Measurement } \\
(\mathrm{cm})\end{array}$ \\
\hline Point 1 & A-1a & 1a-ulnar & $4.0 \pm 0.5$ \\
\cline { 2 - 4 } ECU & & & $8.0 \pm 1.6$ \\
\hline Point 2 & A-2a & 2a-radial & $2.0 \pm 0.4$ \\
\cline { 2 - 4 } ECR & & & $12.0 \pm 1.6$ \\
\hline Point 3 & A-3 & $16.0 \pm 0.5$ \\
EDC & A-4 & $17.0 \pm 0.5$ \\
Point 4 & A-5 & & \\
\hline Point 5 & & & \\
APL & &
\end{tabular}

Muscle coordinates on line A-B including perpendicular ulnar or radial distances for stimulation point 1 and 2 based on normalised forearm length, CI 95\%

$A P L \mathrm{M}$. abductor pollicis longus, $E C U$ M. extensor carpi ulnaris, $E D C$ M. extensor digitorum communis, EPL M. extensor pollicis longus

Furthermore, we presume that the type of lesion might be a predictor for an efficient tenodesis grasp. To prove this presumption and to disclose reproducibility, a standardised testing with ES of the forearm muscles is required. Our mapping of ES of selected muscles makes it feasible to identify whether a LMN or UMN lesion is present. The ES as a diagnostic tool could serve in clinical practice to determine further treatment of the tetraplegic hand.

Anterior horn and cell body damage in the peripheral neuron is present within 8 days after injury. [7, 18]. Damages in the distal cell body cannot immediately be detected. Damaged axons can continue to conduct action potential for almost 8 days after injury [18]. We suggest that the EDC, ECU, EPL and APL that are key muscles for the development of the "functional hand" should be tested by stimulation after 10 days post injury. In case of an UMN lesion in all these muscles a "functional hand" will probably not occur despite of splinting and positioning. Furthermore, prolonged splinting might not lead to better results and could increase the risk of joint contractures. Intrinsic plus splinting for several hours during night time in the first 6 to 8 weeks could be one possibility to avoid intrinsic tightness. The often-used positioning in $30^{\circ}$ wrist extension, $90^{\circ}$ in the MCP and PIP joints and $0^{\circ}$ in the DIP joints during day time could be left. To avoid a forced opening of the hand during activities as wheelchair propelling or physiotherapy activities a tape might be sufficient and could be placed in between the PIP and DIP like a loop.

Muscle-tendon transfer is an established technique to restore hand function in tetraplegia. Typically, muscles of strength grade MRC 4-5 are used as donors. However, even weaker donors (MRC 3) have been successfully transferred in selected cases [19]. It has been suggested that conditioning of muscles via ES prior to surgery would not only improve strength of donor muscles but also facilitate muscle activation and relearning after surgery [20]. Preoperative ES of muscles innervated but weak (MRC 2-3) could potentially expand the number of donors available for transfer.

Another particularly promising treatment option is a nerve transfer to regain finger and thumb extension [21]. Supinator nerve branches are transferred to the posterior interosseous nerve to reinnervate its target muscles. Knowledge of the type of lesion is crucial for timing of surgical intervention after SCI and treatment before surgery. Preoperative ES might help to decide the choice of surgical procedure. If ES is used for diagnostic purpose it could also open a field of treatment before nerve transfer in case of a LMN lesion for the recipient muscles. For example, to provide a satisfying finger extension after nerve transfer contractile elements of the EDC should be preserved. To preserve contractile elements in a muscle with a LMN lesion ES via muscle could be performed, starting directly in the acute phase after SCI, to avoid the structural changes in the muscle due to denervation [10]. Furthermore, the stimulation paradigm, that only muscles with a UMN lesion are eligible for ES should be reconsidered. Kern et al. [22] investigated the effect of ES in denervated muscles. They suggested that nerve regrowth is not inhibited. Furthermore, they observed motor and/or sensory reinnervation under the application of direct muscle stimulation. In another study Salmons and Jarvis [17] could demonstrate in an animal model the therapeutic benefits of an early ES of denervated muscles after injury regarding cross sectional area, fibre size and ultrastructure. The stimulation could be transferred onto the muscles of the upper limbs.

There are limitations in this study. We used surface ES to test the excitability of the muscle to detect the type of lesion. Because of the relatively large electrical field, the accuracy is less than using needle electrodes. However, using surface ES is less invasive, well accepted by patients, feasible in clinical practice and significant enough to get a valid information if the muscle is excitable via nerve. [12, $23,24]$. A neurography in addition could be performed if more information about the peripheral nerve is required.

We mainly concentrated on one clinical observation, namely the presence of a LMN or UMN motor lesion on the EDC and the possible influence of the development of the tenodesis grasp. Of course, other factors as different positioning and treatment strategies as well as spasticity and connective tissue composition might play role in the development of a tenodesis grasp. However, our observation, that we achieved through this retrospective data analysis, might encourage clinicians and therapists to use a feasible ES mapping system as an additional diagnostic tool 
to collect information about the excitability of forearm muscles that influence the tenodesis grasp.

To demonstrate the effectiveness of a positioning and/or splinting procedure on person with tetraplegia with an UMN or LMN larger clinical trials are warranted.

In conclusion, surface ES might serve as a routinely used diagnostic tool in clinical practice to determine the type of lesion in the forearm muscles that are responsible for the development of a tenodesis grasp in patients with tetraplegia. This information offers clues about the expected outcome of the tetraplegic hand regarding form and position.

Funding Funding for this project was provided by the Swiss Paraplegic Centre, Nottwil, Switzerland and University of Gothenburg, Sweden.

\section{Compliance with ethical standards}

Conflict of interest The authors declare that they have no conflict of interest.

\section{References}

1. Doll U, Maurer-Burkhard B, Spahn B, Fromm B. Functional hand development in tetraplegia. Spinal Cord. 1998;36:818-21.

2. MAppSc LH. Principles of conservative management for a nonorthotic tenodesis grip in tetraplegics. J Hand Ther Hanley \& Belfus, Inc. 2012;9:238-42.

3. Thorsen RA, Occhi E, Boccardi S, Ferrarin M. Functional electrical stimulation reinforced tenodesis effect controlled by myoelectric activity from wrist extensors. JRRD. 2006;43:247.

4. Harvey L, Baillie R, Ritchie B, Simpson D, Pironello D, Glinsky $J$. Does three months of nightly splinting reduce the extensibility of the flexor pollicis longus muscle in people with tetraplegia? Physiother Res Int. 2007;12:5-13.

5. Harvey L. Principles of conservative management for a nonorthotic tenodesis grip in tetraplegics. J Hand Ther. 1996;9:238-42.

6. Allieu Y. Surgical management of the adult spastic hand. Chir Main. 2011;30:159-75.

7. Thomas CK, Bakels R, Klein CS, Zijdewind I. Human spinal cord injury: motor unit properties and behaviour. Acta Physiol. 2014;210:5-19.

8. Mulcahey MJ, Smith BT, Betz RR. Evaluation of the lower motor neuron integrity of upper extremity muscles in high level spinal cord injury. Spinal Cord. 1999;37:585-91.

9. Salmons S, Ashley Z, Sutherland H, Russold MF, Li F, Jarvis JC. Functional electrical stimulation of denervated muscles: basic issues. Artif Organs Black Sci Inc. 2005;29:199-202.

10. Boncompagni S, Kern H, Rossini K, Hofer C, Mayr W, Carraro $\mathrm{U}$, et al. Structural differentiation of skeletal muscle fibers in the absence of innervation in humans. Proc Natl Acad Sci USA. 2007;104:19339-44.

11. Mulcahey MJ, Smith BT, Betz RR. Evaluation of the lower motor neuron integrity of upper extremity muscles in high level spinal cord injury. Spinal Cord. 1999;37:585-91.

12. Bryden AM, Hoyen HA, Keith MW, Mejia M, Kilgore KL, Nemunaitis GA. Upper extremity assessment in tetraplegia: The importance of differentiating between upper and lower motor neuron paralysis. Arch Phys Med Rehabil. 2016;97: S97-104.

13. Compston A. Aids to the Investigation of Peripheral Nerve Injuries. Medical Research Council: Nerve Injuries Research Committee. His Majesty's Stationery Office: 1942; p. 48 (iii) and 74 figures and 7 diagrams; with Aids to the Examination of the Peripheral Nervous System. By Michael O'Brien for the Guarantors of Brain. Saunders Elsevier: 2010; p. [8] 64 and 94 Figures. Brain. 2010;133:2838-44. Published by Oxford University Press on behalf of the Guarantors of Brain.

14. Jacobs PL, Nash MS. Exercise recommendations for individuals with spinal cord injury. Sports Med. 2004;34:727-51.

15. Spooren AIF, Janssen-Potten YJM, Kerckhofs E, Bongers HMH, Seelen HAM. ToCUEST: a task-oriented client-centered training module to improve upper extremity skilled performance in cervical spinal cord-injured persons. Spinal Cord. 2011;49:1042-8.

16. Harvey L. Principles of conservative management for a nonorthotic tenodesis grip in tetraplegics. J Hand Ther. 1996;9:238-42.

17. Salmons S, Jarvis JC. Functional electrical stimulation of denervated muscles: an experimental evaluation. Artif Organs. 2008;32:597-603.

18. Miller RG, Peterson C, Rosenberg NL. Electrophysiologic evidence of severe distal nerve segment pathology in the GuillainBarré syndrome. Muscle Nerve. Wiley Subscr Serv, Inc. 1987;10:524-9.

19. Fridén J, Gohritz A, Turcsanyi I, Ejeskär A. Restoration of active palmar abduction of the in te thumb tetraplegia by tendon transfer of the extensor digiti minimi to abductor pollicis brevis. J Hand Surg Eur. 2012;37:665-72.

20. Bersch IDA, Fridén J. Role of functional electrical stimulation in tetraplegia hand surgery. Arch Phys Med Rehabil. 2016;97: S154-9.

21. Bertelli JA, Tacca CP, Ghizoni MF, Kechele PR, Santos MA. Transfer of supinator motor branches to the posterior interosseous nerve to reconstruct thumb and finger extension in tetraplegia: case report. J Hand Surg Eur. 2010;35:1647-51.

22. Kern H, Hofer C, Mödlin M, Forstner C, Raschka-Högler D, Mayr $\mathrm{W}$, et al. Denervated muscles in humans: limitations and problems of currently used functional electrical stimulation training protocols. Artif Organs. 2002;26:216-8.

23. Gobbo M, Maffiuletti NA, Orizio C, Minetto MA. Muscle motor point identification is essential for optimizing neuromuscular electrical stimulation use. J Neuroeng Rehabil BioMed Cent. 2014;11:17.

24. Mulcahey MJ, Smith BT, Betz RR. Evaluation of the lower motor neuron integrity of upper extremity muscles in high level spinal cord injury. Spinal Cord. 1999;37:585-91. 